'Sección Endocrinología y Diabetes. Hospital Clínico de la Universidad de Chile. Santiago, Chile.

${ }^{2}$ Hospital de Medicina Veterinaria de la Universidad de Chile. Santiago, Chile.

${ }^{3}$ Departamento de Cirugía. Hospital Clínico de la Universidad de Chile. Santiago, Chile. ${ }^{4}$ Departamento de Oftalmología. Hospital Clínico de la Universidad de Chile. Santiago, Chile. ${ }^{a}$ Médico veterinario.

Recibido el 13 de septiembre de 2016, aceptado el 4 de abril de 2017.

Correspondencia a: Dra. Alejandra Lanas Santos Dumont 999, Sección de Endocrinología. Independencia. Santiago, Chile. Teléfono: 29788430 alelanasm@gmail.com

\section{Caracterización de pacientes controlados por enfermedad de Basedow Graves en un hospital universitario}

\author{
ALEJANDRA LANAS ${ }^{1}$, PATRICIA DÍAZ ${ }^{1}$, DANIELA EUGENIN ${ }^{1}$, \\ FRANCO GONZÁLEZ ${ }^{2, a}$, PÍA CID ${ }^{1}$, FRANCISCO CORDERO ${ }^{1}$, \\ VERÓNICA ARAYA ${ }^{1}$, CLAUDIO LIBERMAN ${ }^{1}$, MARCELA BARBERAN ${ }^{1}$, \\ PATRICIO GAC ${ }^{3}$, NEIL SALDÍAS ${ }^{4}$, PEDRO PINEDA ${ }^{1}$
}

\section{Clinical features of patients with Basedow Graves disease seen at a university hospital}

Background: Basedow Graves disease (BGD) is the leading cause of hyperthyroidism. The characteristics of patients seen at a university hospital may differ from those described in the general population. Aim: To describe the clinical features of patients with BGD seen at a university hospital. Material and Methods: Review of medical records of all patients seen at our hospital between 2009 and 2014 with the diagnosis of thyrotoxicosis, hyperthyroidism or BGD. Clinical features, laboratory results and treatments were recorded. Results: We reviewed clinical records of 272 patients; 15 had to be excluded due to incomplete data. BGD was present in $77.9 \%(n=212)$. The mean age of the latter was 42 years (range 10-81) and 76\% were women. Ninety six percent were hyperthyroid at diagnosis and thyroid stimulating hormone was below $0.1 \mathrm{mIU} / \mathrm{L}$ in all patients. Median free thyroxin and triiodothyronine levels were $3.26 \mathrm{ng} / \mathrm{dl}$ and $3.16 \mathrm{ng} / \mathrm{ml}$, respectively. Thyrotropin-receptor antibodies were positive in $98.5 \%$ and $85.7 \%$ had positive thyroid peroxidase antibodies. Graves orbitopathy (GO) was clinically present in $55 \%$ of patients. Of this group, $47 \%$ had an active $G O$, $26 \%$ had a moderate to severe disease and $7.8 \%$ had sight-threatening GO. As treatment, $26 \%$ received radioiodine, $44 \%$ anti-thyroid drugs exclusively, $28 \%$ underwent thyroidectomy and $2 \%$ did not require therapy. Conclusions: In this group of patients, we observed a greater frequency of severe eye disease and a high rate of surgical management. This finding could be explained by referral to highly qualified surgical and ophthalmological teams.

(Rev Med Chile 2017; 145: 436-440)

Key words: Graves Disease; Hyperthyroidism; Thyroidectomy.

\section{L} a tirotoxicosis es una condición caracterizada por un excesivo efecto de las hormonas tiroideas a nivel tisular. El hipertiroidismo es el cuadro resultante del exceso de síntesis de hormonas a nivel tiroideo.

En un estudio poblacional, la prevalencia reportada es de $1,3 \%$, correspondiendo $0,7 \%$ a hipertiroidismo subclínico, el cual se define por TSH baja con niveles plasmáticos normales de T3 y $\mathrm{T}^{2}$. La principal causa de hipertiroidismo en regiones con suficiencia de yodo es la enfermedad de Basedow Graves (EBG) ${ }^{3}$. Es una patología tiroidea autoinmune causada por el estímulo de anticuerpos antireceptor de TSH (TRAb) sobre células 
foliculares ${ }^{4}$. Se presenta con mayor frecuencia en mujeres y se asocia a la presencia de orbitopatía distiroídea (OD), clínicamente evidente en 30$50 \%$ de los casos ${ }^{4}$.

Las alternativas terapéuticas más utilizadas son los fármacos antitiroideos (FAT) y el tratamiento con radioyodo, reservándose la cirugía para casos específicos ${ }^{4}$. La elección del tratamiento se basa en aspectos clínicos y condiciones asociadas, como la presencia de OD o el deseo de embarazo, pero también se ha descrito una importante variación geográfica en la aproximación terapéutica. En América Latina, Europa y Asia, la alternativa más utilizada son los FAT, mientras que en Estados Unidos de Norteamérica es la terapia con radioyodo ${ }^{5-6}$. Las características y el tratamiento de los pacientes que se atienden en un centro universitario pueden ser distintas a las descritas en la literatura, por la selección de pacientes de mayor complejidad. El objetivo de este estudio es caracterizar a los pacientes que se controlan por EBG en un centro donde se cuenta con un equipo multidisciplinario especializado para el manejo de esta patología.

\section{Pacientes y Método}

Estudio descriptivo retrospectivo. Se realiza revisión de fichas clínicas de todos los pacientes que se controlan en el Hospital Clínico de la Universidad de Chile con los diagnósticos de: tirotoxicosis, hipertiroidismo o EBG entre los años 2009 y 2014. Se evalúan variables demográficas, características clínicas, etiología, niveles de TSH, T4l, T3, la presencia de TRAb y anticuerpos antitiroperoxidasa (ATPO), la frecuencia de OD y el tratamiento efectuado. Se excluyen del análisis a pacientes con fichas clínicas incompletas respecto al diagnóstico etiológico o al tratamiento efectuado. La EBG fue definida por un médico especialista de acuerdo al cuadro clínico, ecografía tiroidea y captación de radioyodo, siendo confirmado por cintigrama tiroideo y/o la presencia de TRAB positivos. Se realiza cálculo de promedios o de medianas en caso que las variables no presenten distribución normal y se realiza descripción de frecuencias en la población. Para el análisis se utiliza el programa estadístico STATA 10.0.

Este estudio fue aprobado por el Comité de Ética del Hospital Clínico de la Universidad de Chile.

\section{Resultados}

Se revisaron las fichas de 272 pacientes controlados en nuestro centro en el período estudiado. Se excluyeron del análisis 15 pacientes por presentar datos incompletos en la ficha clínica. La etiología más frecuente fue $\mathrm{EBG}$, en $77,9 \%(\mathrm{n}=212)$. Le siguieron en frecuencia el bocio multinodular hiperfuncionante, $12,1 \%(\mathrm{n}=33)$, adenoma tóxico $4,4 \%(n=12)$ y otras causas $5,5 \%(n=15)$.

De los pacientes con EBG, 75,9\% $(n=161)$ correspondían a sexo femenino y la edad promedio fue 41,5 años (rango 10-81 años). El 96,2\% se encontraba hipertiroideo al momento del diagnóstico. En todos estos casos, la TSH fue inferior a 0,1 $\mathrm{mIU} / \mathrm{L}$. La mediana de T4l $=3,26$ (rango $1,2-8,8)$ $\mathrm{ng} / \mathrm{dl}$ y de T3 $=3,16 \mathrm{ng} / \mathrm{mL}$ (rango 1,8-6,8). En 131 pacientes se registró en la ficha el título de TRAb, el que fue positivo en $98,5 \%(n=129)$. En cuanto a los ATPO, se obtuvo el dato en 64 pacientes, resultando positivo en $87,5 \%(\mathrm{n}=56)$ (Tabla 1$)$.

El $54,7 \%(n=116)$ presentaba OD clínicamente evidente. En este grupo, $46,6 \%(n=54)$ correspondió a orbitopatía activa, definido por un índice de actividad CAS $\geq 3^{7}$. En cuanto a la severidad, 25,9\% $(\mathrm{n}=30)$ fue catalogado, de acuerdo a la evaluación oftalmológica, como OD moderada-severa. El 7,8\% $(\mathrm{n}=9)$ presentó riesgo visual, 6 de ellos con neuropatía óptica compresiva, 2 con luxación de globo ocular y uno con úlcera corneal complicada con endoftalmitis (Tabla 2).

La totalidad de estos pacientes fueron evaluados y tratados por un grupo de oftalmólogos expertos en el manejo de la OD. Respecto al tratamiento de los pacientes con OD activa, el tratamiento de primera línea fue con corticoesteroides, indicándose en todos los casos la administración

\section{Tabla 1. Características basales de pacientes con EBG}

\begin{tabular}{|lc|}
\hline Edad (años) (mediana y rango) $(n=212)$ & $41,5(10-81)$ \\
\hline Sexo femenino (\%) & 75,9 \\
T4L $(\mathrm{ng} / \mathrm{dl})$ (mediana y rango) & $3,26(1,2-8,8)$ \\
T3 $(\mathrm{ng} / \mathrm{ml})$ (mediana y rango) & $3,16(1,8-6,8)$ \\
TRAb positivo (\%) & 98,5 \\
\hline ATPO positivo (\%) & 87,5 \\
\hline
\end{tabular}

*El valor $n$ representa el número de pacientes en que se registra en la ficha clínica la variable analizada. 
Tabla 2. Categorización de actividad y severidad de orbitopatía distiroidea en los pacientes con evidencias de compromiso ocular $(n=116)$

\begin{tabular}{|lccc|}
\hline & Activa & Inactiva & \\
Leve & 24 & 53 & $77(66,4 \%)$ \\
Moderada-severa & 21 & 9 & $30(25,9 \%)$ \\
Riesgo visual & 9 & 0 & $9(7,8 \%)$ \\
Total & $54(46,6 \%)$ & $62(53,4 \%)$ & Total $=116$ \\
\hline
\end{tabular}

* Los valores están expresados en número de pacientes y porcentaje del total (\%).

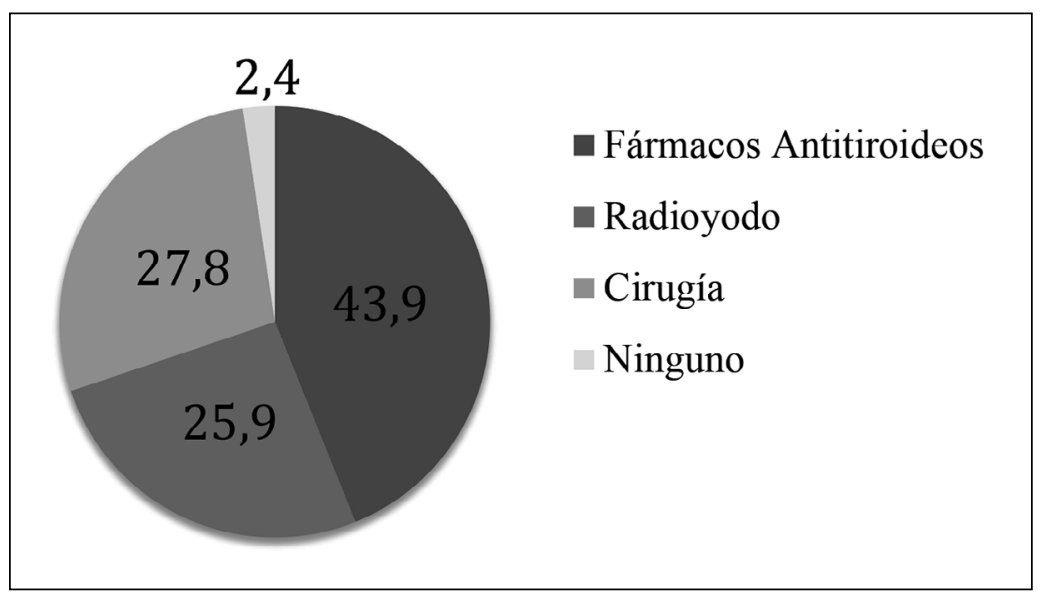

Figura 1. Tratamiento de pacientes con Enfermedad de Basedow Graves en un Centro Universitario. Resultados expresados en porcentaje. endovenosa en bolos de metilprednisolona. Sin embargo, por motivos socioeconómicos o demográficos, en algunos casos no fue posible realizarlo, recibiendo tratamiento con metilprednisolona sólo $60 \%(\mathrm{n}=32)$ y el resto prednisona vía oral.

De los pacientes con OD moderada-severa, 12 requirieron cirugía descompresiva por presentar proptosis severa. En cuanto a los pacientes con OD con riesgo visual que se manifestaron con neuropatía óptica, 2 respondieron de manera favorable al tratamiento con bolos de metilprednisolona y 4 requirieron cirugía descompresiva. Los pacientes con luxación ocular se manejaron con descompresión orbitaria. Todos estos casos evolucionaron de manera favorable, sin observarse casos de pérdida de visión permanente en este período.

Ninguno de los pacientes recibió tratamiento con radioterapia u otra terapia inmunosupresora durante este período.

En cuanto al tratamiento del hipertiroidismo, $25,9 \%(n=55)$ recibió radioyodo, de los cuales
$30,1 \%(\mathrm{n}=17)$ bajo profilaxis con corticoides debido a que presentaban factores de riesgo para OD. El 43,9\% ( $\mathrm{n}=93)$ se manejó exclusivamente con fármacos antitiroideos, en $27,8 \%(\mathrm{n}=59)$ se realizó tiroidectomía y 2,4\% $(n=5)$ no requirieron terapia antitiroidea por mantenerse eutiroideos, hipotiroideos o porque presentaron un hipertiroidismo subclínico transitorio sin factores de riesgo asociados (Figura 1).

Dentro de los motivos de la tiroidectomía, la principal indicación fue la presencia de OD activa $(25,4 \%[\mathrm{n}=15])$, seguido de bocio de gran tamaño o multinodular $(23,7 \%[\mathrm{n}=14])$ y la asociación con cáncer diferenciado de tiroides (CDT) sospechado ecográficamente o confirmado por citología preoperatoria $(23,7 \%[n=14])$. Otras causas registradas fueron la decisión del paciente luego de la no respuesta o recidiva con FAT $(11,9 \%[\mathrm{n}=7])$, reacción adversa a FAT $(8,47 \%[\mathrm{n}=5])$ y deseo de embarazo a corto plazo $(6,78 \%[n=4])$. 
En 6,6\% $(\mathrm{n}=14)$ de los pacientes se diagnosticó cáncer diferenciado de tiroides, siendo 93\% cáncer papilar.

\section{Discusión}

En Chile se realiza yodación de la sal como política de salud pública ${ }^{8}$. En concordancia con lo reportado ${ }^{3}$, en nuestra población, con suficiencia de yodo, la principal causa de hipertiroidismo es la EBG. Las características de los pacientes tratados en nuestro centro respecto a sexo y edad y presencia de anticuerpos son similares a las descritas en la literatura internacional ${ }^{3,4,9}$. La frecuencia encontrada de OD clínicamente evidente es similar a la descrita, pero observamos una mayor presencia de OD activa y de cuadros severos que lo reportado ${ }^{4,7,9,10}$. En concordancia con estos hallazgos, encontramos una alta tasa de cirugía descompresiva orbitaria en el grupo estudiado. Esto puede ser explicado por la existencia de un equipo oftalmológico experto en patología orbitaria, donde se manejan casos de mayor complejidad. El equipo oftalmológico de nuestro centro ha publicado los resultados de pacientes con OD sometidos a descompresión orbitaria en un seguimiento a 9 años, con un buen rendimiento respecto a la disminución del exoftalmos y con ausencia de diplopía inducida por la cirugía ${ }^{11}$. Sin embargo, los datos de otro centro terciario de referencia mostraron una menor frecuencia de casos severos que lo encontrado en nuestro centro ${ }^{12}$.

Las distintas alternativas terapéuticas para la EBG han demostrado una efectividad similar ${ }^{13,14}$, pero presentan algunas diferencias respecto a beneficios en escenarios clínicos particulares y la posibilidad de presentar efectos adversos ${ }^{15,16}$. Los FAT permiten un buen control del hipertiroidismo, pero luego de su suspensión presentan un mayor riesgo de recurrencia con respecto a las otras alternativas terapéuticas ${ }^{14}$.

El radioyodo se asocia a un incremento en el riesgo de OD, lo que se encuentra relacionado a una persistencia de títulos de TRAb elevados, a diferencia de lo observado con FAT y cirugía, en que se observa una disminución de ellos ${ }^{14,17-19}$. Este riesgo es mayor en fumadores, pacientes con T3 elevada y cuando existe hipotiroidismo no controlado después del tratamiento ablativo ${ }^{14,19}$.
La profilaxis con corticoides ha demostrado ser efectiva en prevenir la OD posterior al tratamiento con radioyodo ${ }^{18,19}$.

La cirugía se reserva para casos particulares como bocio de gran tamaño, presencia de nódulos tiroideos sospechosos, falta de respuesta o reacción adversa a FAT en un paciente que rechaza terapia con radioyodo, cuando se planifica un embarazo a corto plazo o por deseo del paciente luego de explicarle las distintas alternativas ${ }^{4,15,16}$. En series quirúrgicas, la tiroidectomía en pacientes con EBG presentó un buen perfil de seguridad con baja tasa de morbilidad ${ }^{20,21}$.

Es por todos estos factores que la elección del tratamiento adecuado depende de las condiciones clínicas individuales y de la decisión del paciente, luego de informarle sobre las distintas alternativas terapéuticas ${ }^{15,16}$.

La terapia más utilizada en nuestro centro fue FAT, concordante a lo reportado en América Latina $^{5,6}$ (Figura 1). Observamos una mayor frecuencia de manejo quirúrgico que lo reportado, pero los motivos para realizar tiroidectomía son los mismos que descritos en la literatura ${ }^{4,15,16}$. La presencia de OD severa o activa fue una causa importante, lo que está en relación a la alta tasa de patología ocular de los pacientes que se controlan en nuestro centro asistencial. La planificación de un embarazo a corto plazo también fue uno de los motivos considerados. Cuando se consultó a especialistas en distintas regiones geográficas sobre el tratamiento de elección en estas dos últimas condiciones, se planteó la tiroidectomía en cerca de $20 \%$ de los casos, una tasa similar a la encontrada en nuestro centro ${ }^{6}$.

La frecuencia de cáncer diferenciado de tiroides en los pacientes en que se realizó tiroidectomía fue $6,6 \%$, similar a la frecuencia de $8-10 \%$ descrita en otras series quirúrgicas, pero que en pacientes seleccionados puede aumentar a $25 \% 0^{20,21}$. Este hallazgo apoya la importancia de realizar una ecografía tiroidea en los pacientes portadores de EBG.

Esta es la mayor serie clínica reportada en nuestro país, reflejando la realidad de un centro donde se realiza un manejo multidisciplinario de esta patología. Existen dos publicaciones previas con número importante de pacientes, pero enfocados principalmente en la efectividad de la terapia del hipertiroidismo $\mathrm{o}^{22,23}$.

La principal limitación de esta investigación es que se obtienen los datos de manera retrospectiva 
desde registros de fichas clínicas, por lo que puede existir algún grado de pérdida de información.

Podemos concluir que las características de los pacientes controlados con EBG en un centro universitario pueden diferir de las descritas en la literatura, por lo que es importante conocer la casuística local para optimizar los recursos y el trabajo del equipo multidisciplinario que maneja a los pacientes con EBG. Las características particulares de la población que es derivada a un centro de alta complejidad pueden explicar la alta frecuencia de oftalmopatía severa y la mayor tasa de manejo quirúrgico.

\section{Referencias}

1. Cooper DS. Hyperthyroidism. Lancet 2003; 362: 459-68.

2. Hollowell JG, Staehling NW, Flanders WD, Hannon WH, Gunter EW, Spencer CA, et al. Serum TSH, T(4), and thyroid antibodies in the United States population (1988 to 1994): National Health and Nutrition Survey (NHANES III). J Clin Endocrinol Metab 2002; 87: 48999.

3. Nyström HF, Jansson S, Berg G. Incidence rate and clinical features of hyperthyroidism in a long-term iodine sufficient area of Sweden (Gothenburg) 2003-2005. Clin Endocrinol (Oxf) 2013; 78 (5): 768-76.

4. Brent GA. Graves' Disease. N Engl J Med 2008; 358: 2594-605.

5. Wartofsky L, Glinoer D, Solomon B, Nagataki S, Lagasse R, Nagayama Y, et al. Differences and similarities in the diagnosis and treatment of Graves' disease in Europe, Japan, and the United States. Thyroid 1991; 1: 129-35.

6. Burch HB, Burman KD, Cooper DS. A 2011 Survey of Clinical Practice Patterns in the Management of Graves' Disease. J Clin Endocrinol Metab 2012; 97: 4549-58.

7. Bartalena L, Baldeschi L, Dickinson AJ, Eckstein A, Kendall-Taylor P, Marcocci C, et al. Consensus statement of the European group on Graves' orbitopathy (EUGOGO) on management of Graves' orbitopathy. Thyroid 2008; 18 (3): 333-46.

8. López Rodríguez G, Muzzo B. Evolución de la nutrición de yodo en la población chilena. Rev Chil Nutr 2006; 33 : 204-6.

9. Weetman AP. Graves' Disease. N Engl J Med 2000; 343 : 1236-48.

10. Bahn RS. Graves' ophthalmopathy. N Engl J Med 2010; 362 (8): 726-38.

11. Saldías N, Torres G, Cárdenas N, García J. Descompresiones orbitarias en orbitopatía tiroidea ¿Rinden todo lo que nos gustaría? Experiencia de 9 años. Archivos Chilenos de Oftalmología 2016. En prensa.

12. Reddy SV, Jain A, Yadav SB, Sharma K, Bhatia E. Prevalence of Graves' ophthalmopathy in patients with Graves' disease presenting to a referral centre in north India. Indian J Med Res 2014; 139 (1): 99-104.

13. Sundaresh V, Brito JP, Wang Z, Prokop LJ, Stan MN, Murad MH, et al. Comparative Effectiveness of Therapies for Graves' Hyperthyroidism: A Systematic Review and Network Meta-analysis. J Clin Endocrin Metab 2013; 98 (9): 3671-7.

14. Törring $O$, Tallstedt $L$, Wallin G, Lundell G, Ljunggren JG, Taube A, et al. Graves' hyperthyroidism: treatment with antithyroid drugs, surgery, or radioiodine-a prospective, randomized study. Thyroid Study Group. J Clin Endocrinol Metab 1996; 81 (8): 2986-93.

15. Bahn RS, Burch HB, Cooper DS, Garber JR, Greenlee MC, Klein I, et al. Hyperthyroidism and other causes of thyrotoxicosis: management guidelines of the American Thyroid Association and American Association of Clinical Endocrinologists. Endocr Pract 2011; 17 (3): 456-520.

16. Burch HB, Cooper DS. Management of Graves Disease A Review. JAMA 2015; 314 (23): 2544-54.

17. Ma C, Xie J, Wang H, Li J, Chen S. Radioiodine therapy versus antithyroid medications for Graves' disease. Cochrane Database Syst Rev 2016; 2: CD010094.

18. Acharya SH, Avenell A, Philip S, Burr J, Bevan JS, Abraham P. Radioiodine therapy (RAI) for Graves' disease (GD) and the effect on ophthalmopathy: a systematic review. Clin Endocrinol (Oxf) 2008; 69 (6): 943-50.

19. Bartalena L. The Dilemma of How to Manage Graves' Hyperthyroidism in Patients with Associated Orbitopathy. J. Clin. Endocrinol. Metab 2011; 96: 592-9.

20. Phitayakorn R, Morales-García D, Wanderer J, Lubitz CC, Gaz RD, Stephen AE, et al. Surgery for Graves' disease: a 25-year perspective. Am J Surg 2013; 206 (5): 669-73.

21. Pradeep PV, Agarwal A, Baxi M, Agarwal G, Gupta SK, Mishra SK. Safety and Efficacy of Surgical Management of Hyperthyroidism: 15-year Experience from a Tertiary Care Center in a Developing Country. World J Surg (2007) 31: 306-12.

22. Pineda $\mathrm{P}$, Michelsen $\mathrm{H}$, Rivera M, Lillo R, Massardo $\mathrm{T}$, Araya $\mathrm{V}$, et al. [Treatment of hyperthyroidism with radioiodine: effects of administered dose on complications and thyroid function]. Rev Med Chile 2000; 128 (5): 499-507.

23. Pineda G, Arancibia P, Mejía G. [Treatment of Basedow-Graves' hyperthyroidism: retrospective analysis after 30 years]. Rev Med Chile 1998; 126 (8): 953-62. 